Brian D. Kent ${ }^{1,2}$

briankent@physicians.ie

@briandkent

\title{
Editorial
}

\section{Targeted therapy in respiratory disease}

Until recently, the management of respiratory disease often relied upon relatively broad and untargeted therapeutic approaches. Patients with severe asthma were by necessity plied with oral corticosteroids (OCS), controlling their airways disease but at the cost of significant medicationrelated morbidity. COPD management was heavily based on lung function measures ahead of other clinical considerations. People with both cystic fibrosis (CF) and non-CF bronchiectasis were given similar combinations of airway clearance techniques and antibiotics. Clinicians managing interstitial lung disease (ILD) clinics had little evidence to guide care of their patients, particularly those with an ILD other than idiopathic pulmonary fibrosis. Things have changed though, and changed dramatically.

The past 20 years have seen parallel revolutions in our understanding of these diseases, in the underlying pathobiological processes driving them, and in the development of treatment approaches tailored to the individual patient and their illness. In this issue of Breathe we explore how patient care has evolved in the management of severe asthma, of CF, of COPD, of non-CF bronchiectasis, and of connective tissue disease (CTD)-related ILD, allowing personalised and targeted treatment to replace the broader brush strokes of the past.

Severe asthma is perhaps the exemplar disease here. The characterisation of the inflammatory pathways underlying asthmatic airways disease has facilitated the development of a growing family of biologic agents with proven ability to improve disease control and to reduce both exacerbation frequency and OCS exposure. Clinicians managing the care of patients with severe asthma now face the pleasant dilemma of having to decide which one of these drugs would best suit each individual. KaVANAGH et al. [1] discuss these therapies and their evidence base, and discuss a framework for choosing the right drug for the right patient. Strides have also been made in personalising COPD care, where it has become clear that a uniform therapeutic approach based on lung function or other severity classifications may not always yield the best outcomes. DuszYK et al. [2] describe how using treatable traits, such as markers of airway inflammation, can be used to guide appropriate treatment in COPD.

Meanwhile, the outlook for people diagnosed with CF has been transformed by the use of cystic fibrosis transmembrane conductance regulator (CFTR)-potentiating medications, designed on the back of advances in understanding of CFTR pathobiology. The use and selection of these agents, and their impact on outcomes, is discussed in depth by TeWKesbury et al. [3]. Non-CF bronchiectasis has for many years been a Cinderella disease in respiratory medicine, with little high-quality laboratory or clinical data available to guide an empirical understanding of patient care. PEMBRIDGE and Chalmers [4] show how our understanding of bronchiectasis has progressed to the point where genuine individualised care for these often frail patients is now possible. Finally, $W u$ and Molyneaux [5] describe how emerging clinical trial data help them select appropriate therapy for their patients with CTD-related ILD.
Cite as: Kent BD. Targeted therapy in respiratory disease. Breathe 2021; 17: 210170.

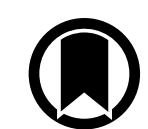

CrossMark 
As a whole, the contents of this issue of Breathe stand as testimony to the contribution of thousands of patients, scientists and clinicians in characterising these diseases, in developing new therapies, and in identifying which new treatments actually work. Huge strides have been made, but the need for further advances is demonstrated in a Patient Voice article describing the successes and frustration associated with receiving biologic therapy for severe asthma [6]. The new treatments we have at our disposal can be transformative for some patients, but are not a universal panacea.

This is my first issue as Chief Editor of Breathe. l'd like to start my 3-year tenure/reign of terror by paying tribute to my immediate predecessor, Prof. Claudia Dobler. Claudia continued the work of previous Breathe editors in making the journal synonymous with high-quality clinical education, and introduced innovative multidisciplinary educational content, such as the popular Lung Function Corner series. At a personal level, she was a great source of advice and guidance, always open to suggestions that could help strengthen the journal. I'd also like to thank the departing members of the Editorial Board for all their work over the past 3 years.

I am delighted to welcome Dr Jayesh Bhatt as Deputy Chief Editor. A veteran Breathe Associate Editor, Jayesh's appointment will allow us to further develop our educational content in paediatric respiratory medicine. Welcome also to our new Associate Editors and ERS Assembly Secretaries, all of whom join the incoming Breathe Editorial Board. I greatly look forward to working with you all to ensure that Breathe remains a benchmark for respiratory clinical education.

Finally, many of you will have read of the recent death of Kai-Håkon Carlsen. Prof. Carlsen held several pivotal roles with the ERS over his long and distinguished career, but also played a key part in the development of an ERS educational journal, which eventually became Breathe. The thoughts of the Breathe editorial team are with his family.

\section{Affiliations}

\section{Brian D. Kent ${ }^{1,2}$}

'Dept of Respiratory Medicine, St James' Hospital, Dublin, Ireland. ${ }^{2}$ School of Medicine, Trinity College Dublin, Dublin, Ireland.

\section{Conflict of interest}

B.D. Kent has participated in advisory boards and/or received speaker fees from Astra Zeneca, Chiesi, GSK, Novartis, and Teva; has received educational travel bursaries from Boehringer Ingelheim, Chiesi, and Napp; and has received research funding from Itamar Medical.

\section{References}

1. Kavanagh JE, Hearn AP, Jackson DJ. A pragmatic guide to choosing biologic therapies in severe asthma. Breathe 2021 in press [https://doi.org/10.1183/20734735.0144-2021]

2. Duszyk K, McLoughlin RF, Gibson PG, et al. The use of treatable traits to address COPD complexity and heterogeneity and to inform the care. Breathe 2021; in press [https://doi.org/ 10.1183/20734735.0118-2021].

3. Tewkesbury DH, Robey RC, Barry PJ. Progress in precision medicine in cystic fibrosis: a focus on CFTR modulator therapy. Breathe 2021; in press [https://doi.org/10.1183/ 20734735.0112-2021]
4. Pembridge T, Chalmers JD. Precision medicine in bronchiectasis. Breathe 2021; 17: 210119.

5. Wu Z, Molyneaux PL. Choosing pharmacotherapy for ILD in patients with connective tissue disease. Breathe 2021; 17: 210114.

6. Denning J, Negus J, Cullen N. Patient experience of biologic treatment for severe asthma: the good, the outstanding and the deeply frustrating. Breathe 2021; in press [https://doi.org/ 10.1183/20734735.0145-2021]. 